\title{
LPV Robust Servo Control of Aircraft Active Side-sticks
}

\author{
Guang Rui Zhou \\ School of Aeronautics and Astronautics, Shanghai Jiao Tong University, Shanghai, China
}

\section{Shi Qian Liu}

School of Aeronautics and Astronautics, Shanghai Jiao Tong University, Shanghai, China and School of Aerospace, Transport and Manufacturing, Cranfield University, Cranfield, UK

\section{Yuan Jun Sang and Xu Dong Wang}

School of Aeronautics and Astronautics, Shanghai Jiao Tong University, Shanghai, China

\section{Xiao Peng Jia}

Department of Basis Research, Chinese Flight Test Establishment, Xi'an, China, and

\section{Er Zhuo Niu}

Department of Basis Research, Xi'an FACRI Aviation Control Technology Co., Ltd, Xi'an, UK

\begin{abstract}
Purpose - This paper aims to focus on the variable stick force-displacement (SFD) gradience in the Active Side Stick (ASS) servo system for the civil aircraft.
\end{abstract}

Design/methodology/approach - The problem of variable SFD gradience was introduced first, followed by the analysis of its impact on the ASS servo system. To solve this problem, a linear-parameter-varying (LPV) control approach was suggested to process variable gradience of the SFD. A $\mathrm{H}_{\infty}$ robust control method was proposed to deal with external disturbance.

Findings - To validate the algorithm performance, a linear time-variant system were calculated to be used to worst cases and the SFD gradience was set to linear and non-linear variation to test the algorithm, and some typical examples of pitch angle and sideslip angle tracking control for a large civil aircraft was also used to verify the algorithm. The results showed that the LPV control method had less settling time and less steady tracking errors than $\mathrm{H}_{\infty}$ control, even in the variable SFD case.

Practical implications - This paper presented an ASS servo system using LPV control method to solve the problem caused by the variable SFD gradience. The motor torque command was calculated by pressure and position feedback without additional hardware support. It was more useful for the electronic hydraulic servo actuator (EHSA).

Originality/value - This was the research paper that analyzed the impact of the variable SFD gradience in the ASS servo system and presented an LPV control method to solve it. It was applicable for the SFD gradience changing in the linear and non-linear cases.

Keywords Flight control, Active side stick, Variable force-displacement gradience, Linear parameter varying system, Robust control, Disturbance rejection. Paper type Research paper. 


\section{Introduction}

Today many aircrafts apply side stick for manipulation because the side stick can supply wide vision during the pilot manipulation and make the pilot comfortable. The traditional passive side stick (PSS) cannot feedback information to the pilot and make them hard feel current flight status of the aircraft. To improve the pilot situation awareness, Hosman first proposed the concept of active side stick (ASS), and the flight state information of the aircraft can be feedbacked to the pilots in the form of stick force. ${ }^{[1]}$ Hanlon developed ASS integration via the double side stick linkage and auto-pilot. ${ }^{[2]}$ After that, the control problem of ASS servo system becomes a hot topic. Feng et al. applied the typical driver model and criteria to study on the pilot induced oscillation (PIO) trend of the different human sense system, and analyzed the influence of the human sense system characteristics on the human-machine coupling plant. ${ }^{[3]} \mathrm{Li}$ et al. used the Hess's structure pilot model to carry out simulating experimental research on the ASS and the PSS and gave the human-machine characteristic evaluation method by the experimental comparison. ${ }^{[4]}$ Liang proposed an adaptive sliding mode control method for the ASS servo system to solve the flutter and disturbance rejection of the side stick. ${ }^{[5]}$ Wei developed an active manipulator and analyze its performance by comparing the steady tracking errors and the control input of the ASS and the PSS under the disturbance. ${ }^{[6]}$

As the ASS has obvious advantages, many researchers make key points to the ASS. Compared with the PSS, ASS applies the servo system to take the place of the spring plant, so the force-displacement gradient value is not constant in the ASS servo system, but varying within some range, that is, force-displacement gradience in the ASS servo system is a bounded time-varying parameter. For the problem of robust control of the system with time-varying parameters, although the traditional proportional integral derivative controller has simple structure and is easy to design and solve it, but it cannot meet the requirement of system robust stability for the system. To solve the problem of robust uncertainty resulted by time-varying parameters and to satisfy the requirement of robust stability of the servo system, a novel robust control method is proposed to design the ASS servo control system.

Motivated by Liang's work, this paper mainly concerns on the ASS servo system with the time-varying force-displacement gradience, and the main contribution of this paper is listed as follows:

1. The quasi-linear-parameter-varying (LPV) models of the ASS servo system with time-varying force-displacement gradience are established;

2. An LPV robust servo control (LPV-RSC) scheme is proposed to solve the problem of the time-varying force-displacement gradience. The proposed LPV-RSC not only stabilizes ASS servo system with external disturbances, but also makes the ASS precisely track the reference inputs even in the worst cases for the force-displacement gradience.

This paper is organized as follows: Section 2 establishes the longitudinal linearization model of a large aircraft and the ASS servo system model; Section 3 gives the controller design of the ASS servo system by using the $\mathrm{H}_{\infty}$ robust control method and the LPVRSC method, respectively. Section 4 carries out some simulations to verify the proposed algorithm. Section 5 summarizes some conclusions. 


\section{Modeling}

\section{Modeling of the active side stick servo system}

The ASS servo system mainly includes the following parts: a motor, a gearbox, mechanical synthesized mechanism and stick, as shown in Figure 1.

Figure 1 ASS dynamics model

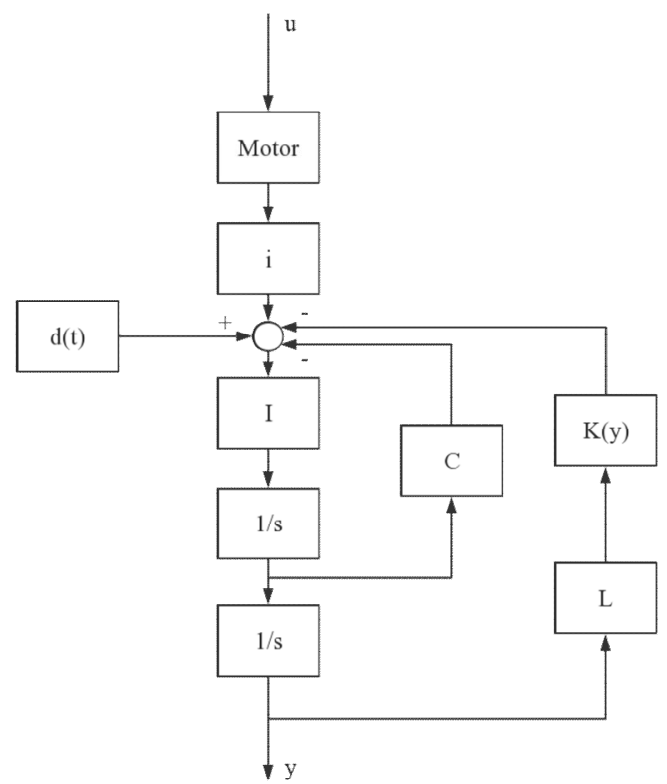

The motor mainly acts as an actuator of the servo system to receive the flight state signal of the aircraft, and the servo control system transforms the signal into the stick displacement of the ASS; The gearbox is directly connected to the motor, it is used to reduce the torque produced by the motor at a certain reduction ratio and then the torque is transmitted to the mechanical synthesized mechanism; the mechanical synthesized mechanism contains a spring, which converts the deceleration torque of the motor into the stick displacement and transmits it to the pilot stick. There are some dynamic relationships between these components and can be expressed as the following differential equation: ${ }^{[5]}$

$$
\begin{gathered}
\ddot{y}(t)=i I u(t)-C I \dot{y}(t)-\operatorname{ILK}(y, t) y(t)+d(t) \\
|d(t)| \leq \Delta \\
|K(y, t)| \leq D
\end{gathered}
$$

where $y$ represents the sick displacement of the ASS, $u$ represents the control input, $d(t)$ represents external interference, and $\Delta$ is its boundary value, $i$ represents the gearbox reduction ratio, $I$ represents the moment of inertia, $C$ represents the friction coefficient, $L$ represents the force arm of the spring force and $K(y, t)$ represents the spring's stiffness coefficient, which is a bounded unknown parameter that varies with time and stick displacement, and $D$ is its boundary value.

In practical applications, the motor of the ASS is generally a permanent magnet three-phase synchronous motor. For simplicity the motor model of the ASS is presented as a second-order system as follows: ${ }^{[6]}$ 


$$
\frac{N_{r e f}(s)}{U(s)}=\frac{\omega_{n m}^{2}}{s^{2}+2 \zeta_{m} \omega_{n m}+\omega_{n m}^{2}}
$$

where $\zeta_{m}, \omega_{n m}$ denote the damping radio and the natural frequency of the motor, $N_{r e f}$ is the reference current signal, $U(s)$ is the motor torque.

And some relevant parameters of the ASS dynamics model are listed in Table $1 .^{[5]}$

Table 1 Related parameters of the ASS dynamics model

\begin{tabular}{cccccc}
\hline \hline$i$ & $I\left(\mathrm{~kg}^{*} \mathrm{~m}^{2}\right)$ & $C$ & $L(\mathrm{~m})$ & $\omega_{n m}(\mathrm{rad} / \mathrm{s})$ & $\zeta_{m}$ \\
\hline $1 / 180$ & 666.67 & 0.15 & 0.05 & 2.5 & 0.7 \\
\hline \hline
\end{tabular}

\section{Aircraft dynamics model}

To verify the tracking performance of the ASS, the quasi-LPV model of the Boeing747 aircraft longitudinal and lateral motions are introduced. ${ }^{[7]}$ The tasks of pitch angle and side-slip angle tracking control are established to illustrate the tracking performance of the ASS. The longitudinal and lateral quasi-LPV models of the aircraft are: ${ }^{[11,14]}$

$$
\begin{aligned}
& \dot{X}_{\text {lon }}=A_{\text {lon }}(\rho) X_{\text {lon }}+B_{\text {lon }}(\rho) U_{\text {lon }} \\
& \dot{X}_{\text {lat }}=A_{\text {lat }}(\rho) X_{\text {lat }}+B_{\text {lat }}(\rho) U_{\text {lat }}
\end{aligned}
$$

where $\quad A_{\text {lon }}(\rho)=A_{\text {lon }, 0}+\rho \cdot A_{\text {lon }, 1}, \quad B_{\text {lon }}(\rho)=B_{\text {lon }, 0}+\rho \cdot B_{\text {lon }, 1}, \quad X_{\text {lon }}=\left[\begin{array}{llll}\Delta u & \Delta \alpha & \Delta \theta & \Delta q\end{array}\right]^{T}, \quad U_{\text {lon }}=\left[\begin{array}{ll}\Delta \delta_{e} & \Delta \delta_{T}\end{array}\right]^{T}$, $A_{\text {lat }}(\rho)=A_{\text {lat }, 0}+\rho \cdot A_{\text {lat }, 1}, B_{\text {lat }}(\rho)=B_{\text {lat }, 0}+\rho \cdot B_{\text {lat }, 1}, X_{\text {lat }}=\left[\begin{array}{llll}\Delta \beta & \Delta p & \Delta r & \Delta \phi\end{array}\right]^{T}, U_{\text {lat }}=\left[\begin{array}{ll}\Delta \delta_{a} & \Delta \delta_{r}\end{array}\right]^{T}, \rho=V$.

The elevator and throttle are selected as the actuators for the longitudinal motion of the aircraft, which can be represented by the first-order transfer function as equation (7a) and Eq. (7b). The aileron and rudder are also selected as the actuators for the lateral motion of the aircraft, which can be represented by the second-order transfer functions as equation (7c) and Eq. (7d).

For the elevator, the deflection ranges from $-23^{\circ}$ to $17^{\circ}$ and the rotation rate varies from $-37^{\circ} / s$ to $37^{\circ} / s$. Therefore, its transfer function is expressed as: ${ }^{[12]}$

$$
\frac{\Delta \delta_{e}(s)}{\delta_{e_{C}}(s)}=\frac{37}{s+37}
$$

where $\delta_{e_{c}}$ is the elevator command, $\Delta \delta_{e}$ is the actual elevator deflection.

For the throttle, the maximum input is $167000 \mathrm{~N}$ and the maximum change rate is $83500 \mathrm{~N} / \mathrm{s}$. Therefore, its transfer function is expressed as: ${ }^{[12]}$

$$
\frac{\Delta \delta_{T}(s)}{\delta_{T_{C}}(s)}=\frac{0.5}{s+0.5}
$$

where $\delta_{T_{C}}$ is the throttle command, $\Delta \delta_{T}$ is the actual throttle deflection.

For the aileron, the deflection ranges from $-25^{\circ}$ to $15^{\circ}$ and the rotation rate varies form $-55^{\circ} / \mathrm{s}$ to $45^{\circ} / \mathrm{s}$. Therefore, its transfer function is expressed as: ${ }^{[14]}$ 


$$
\frac{\Delta \delta_{a}(s)}{\delta_{a_{c}}(s)}=\frac{-1}{0.0037 s^{2}+0.0821 s+1}
$$

where $\delta_{a_{c}}$ is the aileron command, $\Delta \delta_{a}$ is the actual aileron deflection.

For the rudder, the deflection ranges from $-25^{\circ}$ to $25^{\circ}$ and the maximum change rate is $50^{\circ} / \mathrm{s}$. Therefore, its transfer function is expressed as: $:^{[14]}$

$$
\frac{\Delta \delta_{r}(s)}{\delta_{r_{c}}(s)}=\frac{-1}{0.00198 s^{2}+0.143 s+1}
$$

where $\delta_{r_{c}}$ is the aileron command, $\Delta \delta_{r}$ is the actual aileron deflection.

\section{The servo system controller design}

The ASS servo system is actually a linear parameter varying system, so the robust stability of the system is considered here.

\section{$H_{\infty}$ robust controller design of the active side stick}

The essence of the $H_{\infty}$ robust control problem is to transform the problem of the $H_{\infty}$ robust control into an optimization problem ${ }^{\text {[13] }}$, which can guarantee the initial stability of the closed-loop system and the $H_{\infty}$ norm of the closed-loop system is smaller than the prescribed parameter $\gamma$. The $H_{\infty}$ controller of the ASS can be designed as:

$$
\left[\begin{array}{c}
\dot{x}_{k} \\
u
\end{array}\right]=\left[\begin{array}{ll}
A_{k} & B_{k} \\
C_{k} & D_{k}
\end{array}\right]\left[\begin{array}{c}
x_{k} \\
y
\end{array}\right]
$$

where $x_{k}$ is the state variable of the controller, $A_{k}, B_{k}, C_{k}, D_{k}$ are the state space matrixes of the controller, and $u$ is the control output of the controller, $y$ is the measurement output of the ASS.

There are many methods for solving the $H_{\infty}$ optimization problem, ${ }^{[8,9,10]}$ and the linear matrix inequality (LMI) method is used here. Consider a linear time invariant plant $P$ given by:

$$
\left[\begin{array}{l}
\dot{x} \\
z \\
y
\end{array}\right]=\left[\begin{array}{ccc}
A & B_{1} & B_{2} \\
C_{1} & D_{11} & D_{12} \\
C_{2} & D_{21} & D_{22}
\end{array}\right]\left[\begin{array}{l}
x \\
w \\
u
\end{array}\right]
$$

where all matrices as of $A, B_{1}, B_{2}, C_{1}, C_{2}, D_{11}, D_{12}, D_{21}, D_{22}$ have appropriate dimensions, $x$ denotes the state variables of the plant, $w$ denotes the disturbance input of the plant, $u$ denotes the control variables of the plant, $z$ denotes the controllable output variables of the plant, $y$ denotes the measurable output variables of the plant.

According to ${ }^{[11]}$, a set of LMIs as follows,

$$
\begin{gathered}
\left(\begin{array}{cc}
N_{R} & 0 \\
0 & I
\end{array}\right)^{T}\left(\begin{array}{ccc}
A R+R A^{T} & R C_{1}^{T} & B_{1} \\
C_{1} R & -\gamma I & D_{11} \\
B_{1}^{T} & D_{11}^{T} & -\gamma I
\end{array}\right)\left(\begin{array}{cc}
N_{R} & 0 \\
0 & I
\end{array}\right)<0 \\
\left(\begin{array}{cc}
N_{S} & 0 \\
0 & I
\end{array}\right)^{T}\left(\begin{array}{ccc}
A^{T} S+S A & S B_{1} & C_{1}^{T} \\
B_{1}^{T} S & -\gamma I & D_{11}^{T} \\
C_{1} & D_{11} & -\gamma I
\end{array}\right)\left(\begin{array}{cc}
N_{S} & 0 \\
0 & I
\end{array}\right)<0 \\
\left(\begin{array}{ll}
R & I \\
I & S
\end{array}\right) \geq 0
\end{gathered}
$$


where $N_{\mathrm{R}} \in \mathrm{R}^{\mathrm{n} \times \mathrm{m}}$ and $N_{\mathrm{S}} \in \mathrm{R}^{\mathrm{n} \times \mathrm{m}}$ is a null space of the matrices $\left[B_{2}^{\mathrm{T}} D_{12}{ }^{\mathrm{T}} 0\right]$ and $\left[C_{2} D_{21} 0\right]$, respectively. Therefore, the timecontinuous $\gamma$ optimization problem is solved if and only if there exist a pair of matrices $(R, S)$ satisfying the LMIs of equation (10). Finally, the $H_{\infty}$ controller $K(s)$ can be constructed by using the matrices $R, S$ and $\gamma$.

To facilitate to convert the $H_{\infty}$ controller solution problem of the ASS servo system into the $H_{\infty}$ optimization problem, a generalized system is proposed and its structure is as Figure 2.

Figure 2 Generalized ASS system structure diagram

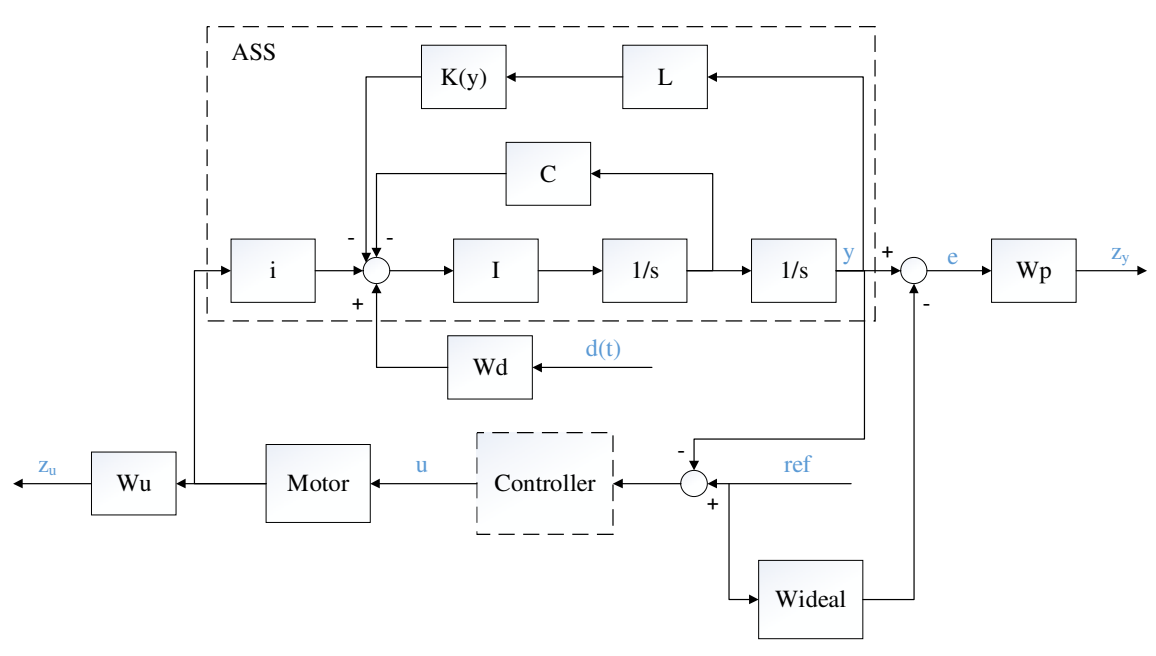

where $W_{\text {ideal }}$ is the ideal model weighting function, $W_{p}$ denotes the performance weighting function, $W_{u}$ is the control input weighting function and $W_{d}$ is the disturbance weighting function.

The disturbance weighting function $W_{d}$ is used to estimate the magnitude of the disturbance and the normalization process is used to reduce the influence of external interferences on the system. Usually, the external interference is relatively small, so $W_{d}=1$ is generally adopted. ${ }^{[15]}$

The control input weighting function $W_{u}$ is used to regulating the control input of the system. $W_{u}$ is chosen as follows: ${ }^{[16]}$

$$
W_{u}=\frac{1}{u_{\max }}
$$

where $u_{\max }$ is the maximum output of the actuator. As the maximum torque output from the motor is $50 \mathrm{~N} \cdot \mathrm{m}, \mathrm{set} \quad u_{\max }=50$.

The performance weighting function $W_{p}$ is used to control the error between the actual output and the ideal output. To ensure that the system steady-state error is small within the system bandwidth, the $W_{p}$ is generally as follows: ${ }^{[17]}$

$$
W_{p}=\frac{\frac{1}{M} s+\omega_{b}}{s+\omega_{b} A}
$$

where $M$ sensitivity peak; $\omega_{b}$ is the desired closed-loop system bandwidth; $A<1$ is the minimum allowed steady state offset. To make the closed-loop system have the appreciate truncation frequency and bandwidth, choose $M=1.3, \omega_{b}=5 \mathrm{rad} / \mathrm{s}$ and $A=0.001$.

The ideal model weighting function $W_{\text {ideal }}$ provides the desired damping ratio and bandwidth of the closed-loop system. $W_{\text {ideal }}$ is usually represented by a first-order transfer function or a second-order one as follows: ${ }^{[18]}$

$$
W_{\text {ideal }}=\left\{\begin{array}{c}
\frac{1}{\tau s+1} \\
\frac{\omega^{2}}{s^{2}+2 \xi \omega s+\omega^{2}}
\end{array}\right.
$$


where tau denotes constant value of time $W_{\text {ideal }}$ is selected as the second-order transfer function with the damping ratio $\xi=0.7$ and the natural frequency $\omega=2.5 \mathrm{rad} / \mathrm{s}$.

The above weighting functions are listed as Table 2 .

Table 2 Selection of weighting functions in $H_{\infty}$ control design

\begin{tabular}{|c|c|c|}
\hline Description & Symbol & Expression \\
\hline External disturbance & $W_{d}$ & $1 \mathrm{rad}$ \\
\hline Control Effort & $W_{u}$ & $\frac{1}{50} N \cdot m$ \\
\hline Performance & $W_{p}(s)$ & $\frac{0.909 s+5}{s+0.005}$ \\
\hline Ideal Command & $W_{\text {ideal }}(s)$ & $\frac{6.25}{s^{2}+3.5 s+6.25}$ \\
\hline
\end{tabular}

After the appropriate weighting functions are selected, the mathematical tool can be used to solve the $H_{\infty}$ optimization problem. In this paper, the robust control toolbox in MATLAB is used to solve the LMIs. Using the optimization solver mincx $(\bullet)$ function, the smallest value of $\gamma$ meeting the LMI (10) can be found. Then the robust controller $K(s)$ is constructed by the optimized solutions $R, S$ and $\gamma$ of the LMIs (10).

\section{Design of the linear-parameter-variable controller}

According to the ASS dynamics, the ASS servo system is a linear parameter varying system whose stick force-to-displacement gradient is a time-varying parameter. Therefore, before using the LPV control method to design the controller, the quasi-LPV model of the ASS servo system is established. ${ }^{[19]}$

The time-varying parameter in the ASS servo system is the stick force-to-displacement gradient of $K$. So the stick force-todisplacement gradience of $K(y, t)$ is selected as the time-varying parameter $\rho$ of the quasi-LPV model for the ASS, that is, scheduling parameter $\rho(t)=K(y, t)$. Denote $x=\left[\begin{array}{l}y \\ \ddot{y}\end{array}\right], w=\left[\begin{array}{c}d \\ y_{r e f}\end{array}\right]$. The state space form of the ASS dynamics model is as follows:

$$
\begin{gathered}
\ddot{x}=\left[\begin{array}{cc}
0 & 1 \\
-I L \rho & -C I
\end{array}\right] x+\left[\begin{array}{ll}
0 & 0 \\
1 & 0
\end{array}\right] w+\left[\begin{array}{l}
0 \\
i I
\end{array}\right] u \\
z=\left[\begin{array}{l}
Z_{y} \\
z_{u}
\end{array}\right]=\left[\begin{array}{cc}
W_{p} & 0 \\
0 & 0
\end{array}\right] x+\left[\begin{array}{cc}
0 & W_{p} \\
0 & 0
\end{array}\right] w+\left[\begin{array}{c}
0 \\
W_{u}
\end{array}\right] u \\
e=y-y_{\text {ref }}=\left[\begin{array}{ll}
1 & 0
\end{array}\right] x-\left[\begin{array}{ll}
0 & 1
\end{array}\right] w
\end{gathered}
$$

Denote:

$$
\begin{gathered}
A(\rho)=\left[\begin{array}{cc}
0 & 1 \\
-I L \rho & -C I
\end{array}\right], B_{1}=\left[\begin{array}{ll}
0 & 0 \\
1 & 0
\end{array}\right], B_{2}=\left[\begin{array}{c}
0 \\
i I
\end{array}\right], C_{1}=\left[\begin{array}{cc}
W_{p} & 0 \\
0 & 0
\end{array}\right], C_{2}=\left[\begin{array}{ll}
1 & 0
\end{array}\right] \\
D_{11}=\left[\begin{array}{cc}
0 & W_{p} \\
0 & 0
\end{array}\right], D_{12}=\left[\begin{array}{c}
0 \\
W_{u}
\end{array}\right], D_{21}=\left[\begin{array}{ll}
0 & 1
\end{array}\right], D_{22}=0
\end{gathered}
$$

where $W_{p}$ and $W_{u}$ are the associated state-space terms of the weighting functions of $W_{p}(s)$ and $W_{u}(s)$, respectively, $\rho$ represents the force-displacement gradient. 
As the time-varying parameter $\rho$ is only included in the state space matrix $A$, the matrix $A$ is expressed in the form affine, that is,

[20]

$$
A(\rho)=A_{0}+\rho A_{1}
$$

where, $A_{0}=\left[\begin{array}{cc}0 & 1 \\ 0 & -C I\end{array}\right], \quad A_{1}=\left[\begin{array}{cc}0 & 0 \\ -I L & 0\end{array}\right]$. So we get the quasi-LPV model of the ASS dynamics model.

The design process of the LPV controller has many similarities with that of the $H_{\infty}$ robust controller, but there are some differences:

1. The number of LMIs that need to be solved is different. As the state space matrix of the LPV system changes with the parameter $\rho$ continuously at any time. In theory, the comprehensive condition of the LMIs equation (10) must contain every value in the feasible domain of the time-varying parameter, which makes the problem become a problem for solving infinite LMIs. Therefore, in practical problems, the "grid" method is usually used to divide the feasible domain of the time-varying parameter, so that the $n$ separation points obtained by the division are used to replace the dynamic model of the entire parameter region. Thus, the LMI problem defined in equation (10) can be expressed as the following $3 n$ LMIs. ${ }^{[21]}$

$$
\begin{gathered}
\left(\begin{array}{cc}
\widehat{N}_{R_{i}} & 0 \\
0 & I
\end{array}\right)^{T}\left(\begin{array}{ccc}
\hat{A}_{i} \hat{R}_{i}+\hat{R}_{i} \hat{A}_{i}^{T} & \hat{R}_{i} \hat{C}_{1_{i}}^{T} & \hat{B}_{1_{i}} \\
\hat{C}_{1_{i}} \hat{R}_{i} & -\gamma I & \widehat{D}_{11_{i}} \\
\hat{B}_{1_{i}}^{T} & \widehat{D}_{11_{i}}^{T} & -\gamma I
\end{array}\right)\left(\begin{array}{cc}
\widehat{N}_{R_{i}} & 0 \\
0 & I
\end{array}\right)<0 \\
\left(\begin{array}{cc}
\widehat{N}_{S_{i}} & 0 \\
0 & I
\end{array}\right)^{T}\left(\begin{array}{ccc}
\hat{A}_{i}^{T} \hat{S}_{i}+\hat{S}_{i} \hat{A}_{i} & \hat{S}_{i} \hat{B}_{1 i} & \hat{C}_{1_{i}}^{T} \\
\hat{B}_{1 i}^{T} \hat{S}_{i} & -\gamma I & \widehat{D}_{11_{i}}^{T} \\
\hat{C}_{1_{i}} & \widehat{D}_{11_{i}} & -\gamma I
\end{array}\right)\left(\begin{array}{cc}
\widehat{N}_{S_{i}} & 0 \\
0 & I
\end{array}\right)<0 \\
\left(\begin{array}{cc}
\hat{R}_{i} & I \\
I & \hat{S}_{i}
\end{array}\right) \geq 0
\end{gathered}
$$

where $\hat{A}_{i}=A_{0}+A_{1} \cdot \rho_{i}, \hat{B}_{1_{i}}=B_{1_{0}}+B_{1_{1}} \cdot \rho_{i}, \hat{C}_{1_{i}}=C_{1_{0}}+C_{1_{1}} \cdot \rho_{i}, \widehat{D}_{11_{i}}=D_{11_{0}}+D_{11_{1}} \cdot \rho_{i}$, for $i=1,2, \cdots, n . \hat{R}_{i}$ and $\hat{S}_{i}$ can be expressed as $\hat{A}_{i}$ for the matrices of $R(\rho)$ and $S(\rho)$.

2. The method of constructing the controller is different. For the $\mathrm{H}_{\infty}$ robust control method, it is only necessary to construct a separate controller by solving LMIs at a single design point. For the LPV control method, the LMIs constructed by all linear state space models are needed and then the integrated $R(\rho)$ and $S(\rho)$ matrices are solved to construct the controller $K(\rho)$. Therefore, to improve the performance of the resulting controller, it is necessary to find a series of $R(\rho)$ and $S(\rho)$ matrices such that the matrix variables $R(\rho)$ and $S(\rho)$ can be expressed linearly as a function of the time-varying parameter $\rho$, as follows: ${ }^{[22]}$

$$
\begin{gathered}
R(\rho)=R_{0}+\rho R_{1} \\
S(\rho)=S_{0}+\rho S_{1}
\end{gathered}
$$

So the controller constructed by $R(\rho)$ and $S(\rho)$ can achieve optimal performances at each working point in the flight envelope while meet all LMI constraints, and it can guarantee the stability of the control system at all working points.

Based on the above two points, the corresponding LPV controller can be obtained through the function hinfgs $(\bullet)$ in Matlab robust tool box after selecting the appropriate weighting function, which refer to equation (11)-(13). To make the LPV controller have 
better performances, we regulate the parameters of each weighting function. Let $M=1.1, \omega_{b}=0.35, A=0.001$. In addition, the sensor noise weighting function $W_{n}$ is selected to reduce the influence caused by sensor noises. The $W_{n}$ is generally as follows:

$$
W_{n}=\frac{s+\omega_{n}}{B s+\omega_{n} C}
$$

where $B$ and $C$ represent the frequency range of the noise signal to be reduced, $B$ is the lower bound, $C$ is the upper bound; $\omega_{n}$ is the bandwidth of the noise signal. So choose $B=0.01, C=100, \omega_{n}=0.4 \mathrm{rad} / \mathrm{s}$.

Table 3 lists each weighting function of the LPV controller design.

Table 3 The weighting functions in LPV control design

\begin{tabular}{ccc}
\hline \hline Description & Symbol & Expression \\
\hline External disturbance & $W_{d}$ & $1 \mathrm{rad}$ \\
Control Effort & $W_{u}$ & $\frac{1}{50} \mathrm{~N} \cdot \mathrm{m}$ \\
Performance & $W_{p}(s)$ & $\frac{0.909 s+0.35}{s+0.00035}$ \\
Ideal Command & $W_{\text {ideal }}(s)$ & $\frac{6.25}{s^{2}+3.5 s+6.25}$ \\
Sensor Noise & $W_{n}(s)$ & $\frac{s+0.4}{0.01 s+400}$ \\
\hline \hline
\end{tabular}

\section{Numerical simulation experiments}

The task is to track pitch angle signal of the aircraft's longitudinal and the side slip angle of the lateral motion. The current test models of the pilot and the aircraft are numerical; the further verification could be implemented based on the physical ASS bench or the real aircraft. To simulate the input stick force of the pilot, the Hess structure pilot model is introduced, as shown in Figure 3. [23]

Figure 3 Hess structure pilot model

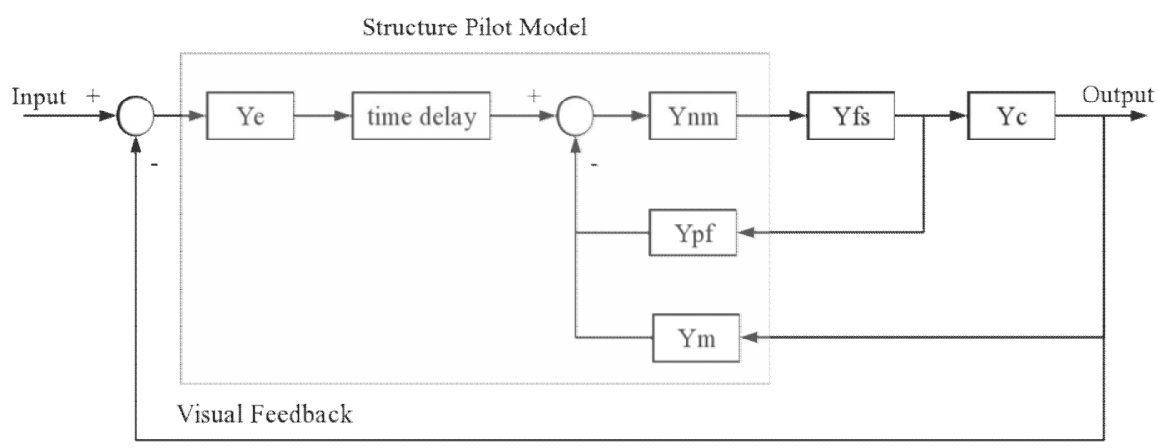

As can be seen from Figure 3, the Hess structure pilot model mainly includes four parts: visual gain $\mathrm{Y}_{\mathrm{e}}$, time delay TD, neuromuscular model $\mathrm{Y}_{\mathrm{nm}}(s)$, and personal feeling feedback $\mathrm{Y}_{\mathrm{pf}}(s) . \mathrm{Y}_{\mathrm{m}}(\mathrm{s})$ denotes the vestibule feedback. $\mathrm{Y}_{\mathrm{c}}(\mathrm{s})$ denotes the plant to be controlled. Table 4 lists the transfer function expressions for the four parts of the Hess structure pilot model. ${ }^{[24]}$

Table 4 Transfer function of the Hess structure pilot model 


\begin{tabular}{ccc}
\hline \hline Description & Symbol & Expression \\
\hline Visual Gain & $\mathrm{Ye}_{\mathrm{e}}$ & 120 \\
Time Delay & $\mathrm{TD}$ & $0.2 \mathrm{~s}$ \\
Neuromuscular Model & $\mathrm{Ynm}_{\mathrm{nm}}(s)$ & $\frac{100}{s^{2}+14 s+100}$ \\
Personnel Feeling & $\mathrm{Ypr}_{\mathrm{pf}}(s)$ & $\frac{3.48}{s+1}$ \\
Feeling System & $\mathrm{Y}_{\mathrm{fs}}(s)$ & $\frac{676}{s^{2}+31.2 s+676}$ \\
\hline \hline
\end{tabular}

The flight situation is at the speed from $187.4 \mathrm{~m} / \mathrm{s}$ to $312.3 \mathrm{~m} / \mathrm{s}$ and altitude of $7000 \mathrm{~m}$, the longitudinal and lateral quasi-LPV model of the large aircraft can be presented in the multitopic form as follow:

$$
\begin{aligned}
& A_{\text {lon }}(\rho)=\left[\begin{array}{cccc}
-0.0062 & 5.0198 & -9.7850 & -0.1176 \\
-0.0006 & -0.4339 & 0 & 1.0027 \\
0 & 0 & 0 & 1.0000 \\
0.0001 & -0.8579 & 0 & -0.5283
\end{array}\right] \rho_{1}+\left[\begin{array}{cccc}
-0.0986 & 5.4133 & -9.7850 & -0.1178 \\
-0.0006 & -0.3259 & 0 & 1.0049 \\
0 & 0 & 0 & 1.0000 \\
0.0059 & -1.1307 & 0 & -0.7780
\end{array}\right] \rho_{2} \\
& B_{\text {lon }}(\rho)=\left[\begin{array}{cc}
0 & 0 \\
0.0424 & 0.0715 \\
0 & 0 \\
1.6944 & 2.7231
\end{array}\right] \rho_{1}+\left[\begin{array}{cc}
0 & 0 \\
0.0263 & 0.1046 \\
0 & 0 \\
1.5117 & 6.7286
\end{array}\right] \rho_{2} \\
& A_{\text {lat }}(\rho)=\left[\begin{array}{cccc}
-0.0824 & 0.0775 & -0.9931 & 0.0521 \\
-2.4483 & -0.6573 & 0.3229 & -0.0002 \\
0.6269 & -0.0548 & -0.1312 & -0.0013 \\
0 & 1 & 0.0761 & 0
\end{array}\right] \rho_{1}+\left[\begin{array}{cccc}
-0.1076 & -0.0155 & -0.9968 & 0.0313 \\
0.2839 & -0.8353 & -0.0686 & -0.00003 \\
1.9129 & -0.0056 & -0.1482 & -0.0026 \\
0 & 1 & -0.0159 & 0
\end{array}\right] \rho_{2} \\
& B_{\text {lat }}(\rho)=\left[\begin{array}{cc}
0 & 0.0065 \\
0.1531 & 0.0915 \\
0.0156 & -0.2401 \\
0 & 0
\end{array}\right] \rho_{1}+\left[\begin{array}{cc}
0 & 0.0004 \\
0.1663 & -0.0039 \\
-0.0499 & -0.0741 \\
0 & 0
\end{array}\right] \rho_{2}
\end{aligned}
$$

where $\theta_{1}=\frac{V-\underline{V}}{\bar{V}-\underline{V}} ; \theta_{2}=\frac{\bar{V}-V}{\bar{V}-\underline{V}} ; \bar{V}$ is the upper bound of $V ; \underline{V}$ is the lower bound of $V$.

As the relationship between the stick force-displacement (SFD) gradience and the stick displacement in the ASS is unknown, three different cases, which represent three common relationship of constant, linear and non-linear are studied. In these cases, the task is to track the pitch angle and the side slip angle by using the two control strategies mentioned in the previous section. The civil aircraft's ASS human-in-the-loop system used in the simulation is shown as Figure 4. There are two closed loops in the system. The inner loop is the stick force tracking loop. The ASS control system synthesizes the flight status feedback by the aircraft and the stick force and outputs the current signal to the motor. The motor drives the side stick to finish the tracking control of the stick force. The outer loops are the pitch angle and the side slip angle tracking loop. The errors between the outputs of the aircraft and the command inputs form the control and drive the outer loop of the overall system. Simulation results are shown as follows. Table 5 lists the relationship between the stick force-displacement gradience and the stick displacement in different cases.

Figure 4 Civil aircraft's active side-stick human-in-the-loop system 


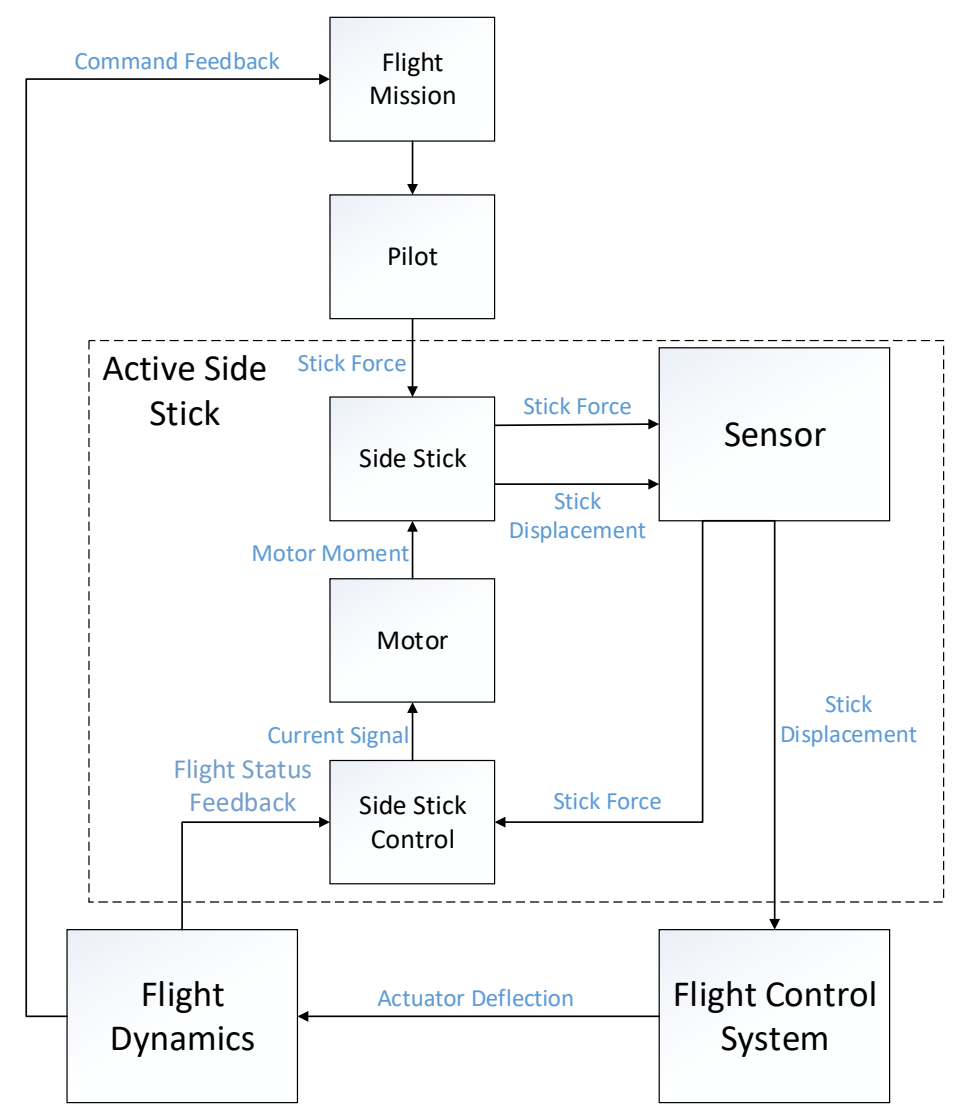

Table 5 Statement of three different simulation cases

\begin{tabular}{ccc}
\hline \hline Case & Expression & Disturbance \\
\hline Case 1 & $K(y, t)=7.5 N / \mathrm{rad}$ & $d(t)=0.1 \sin (10 * t)$ \\
Case 2 & $K(y, t)=7.5 * y+7.5$ & $d(t)=0.1 \sin (10 * t)$ \\
Case 3 & $K(y, t)=7.5 * \sin (y)+7.5$ & $d(t)=0.1 \sin (10 * t)$ \\
\hline \hline
\end{tabular}

\section{Case 1}

When the force-displacement gradient is a constant value, the simulation results of the $H_{\infty}$ and LPV control strategies are shown in Figure 5. Figure 5(a) shows the pitch angle tracking response under the step signal input, Figure 5(b) is the pitch angle tracking error and Figure 5(c) is the pitch motor torque output. Figure 5(d) shows the side-slip angle tracking response under the step signal input, Figure 5(e) is the side-slip angle tracking error and Figure 5(f) is the side-slip motor torque output.

Figure 5 Tracking responses of pitch angle and side-slip angle step input. 


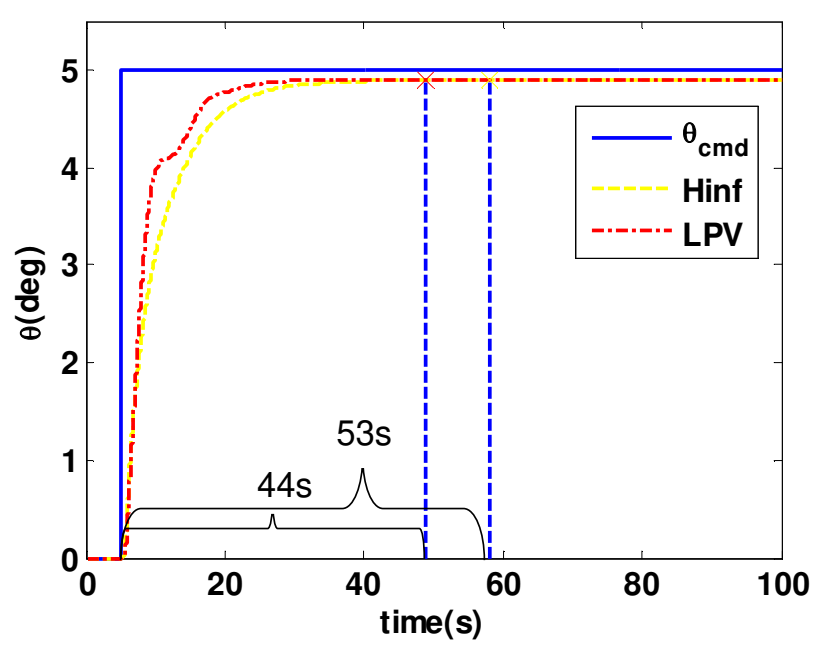

(a)

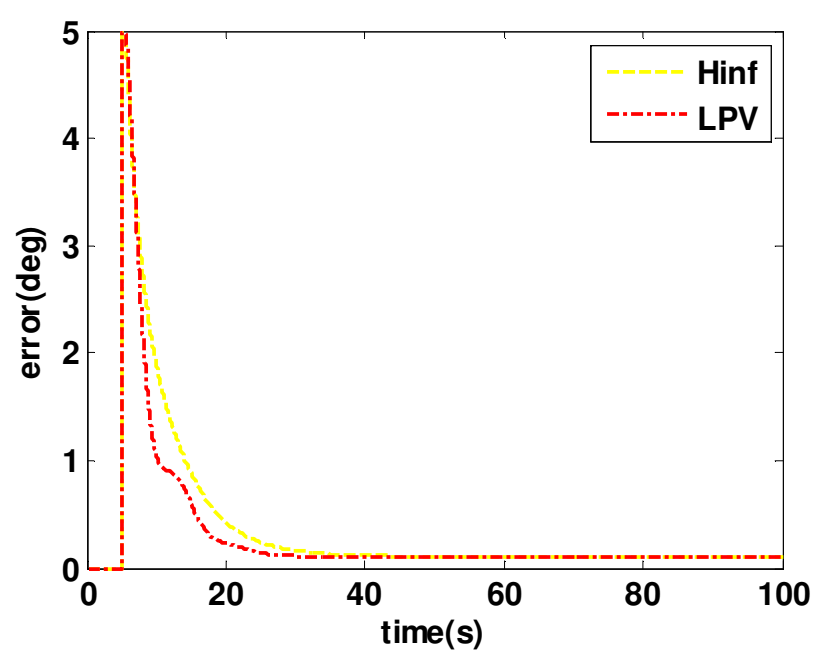

(b)

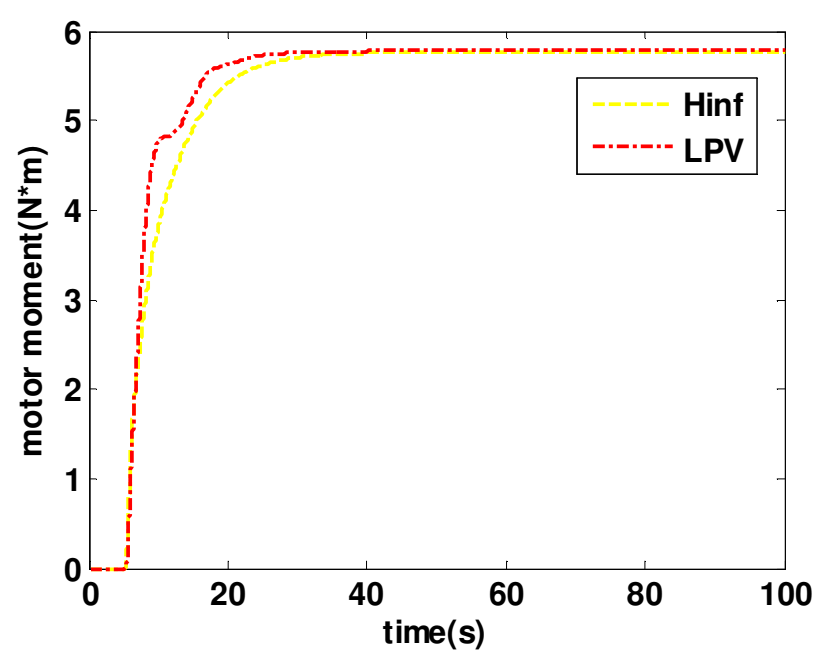

(c)

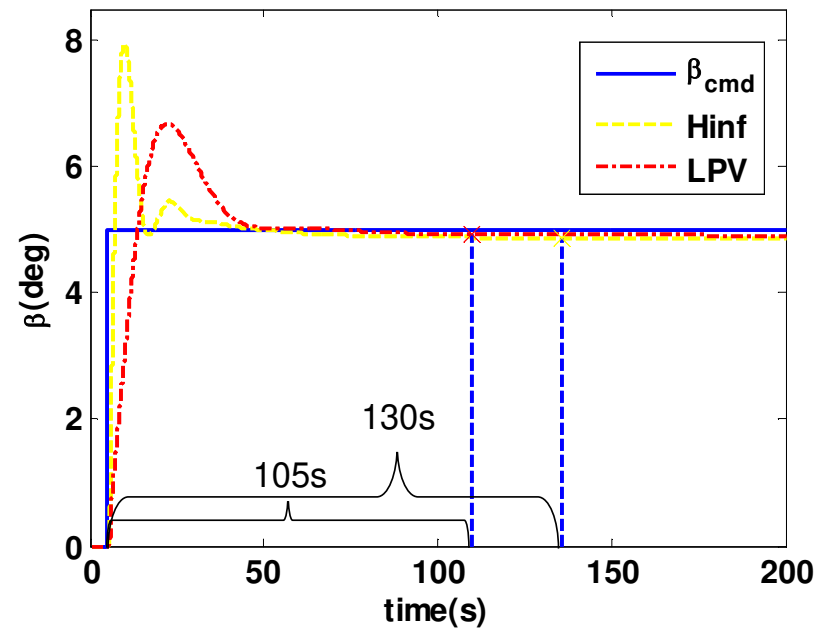

(d)

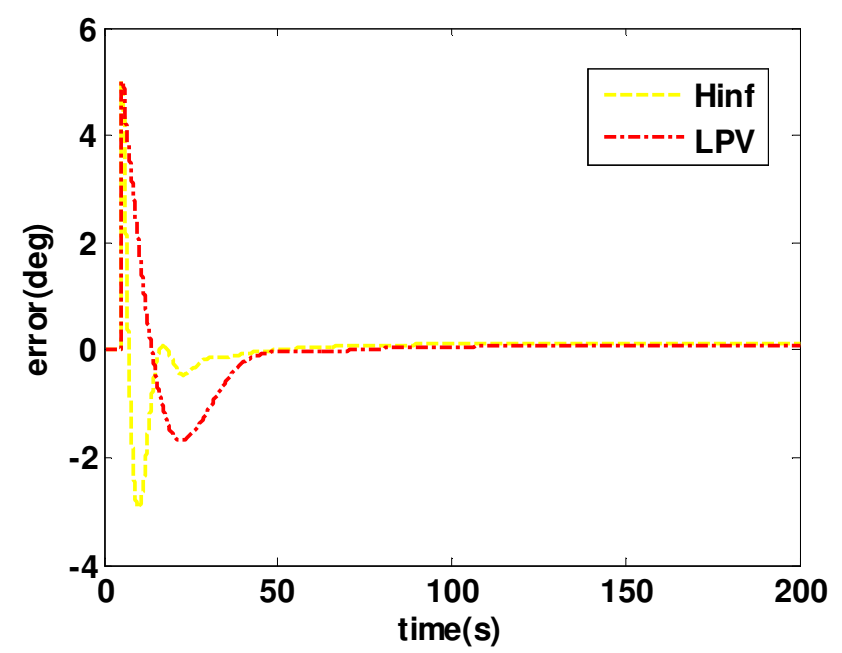

(e)

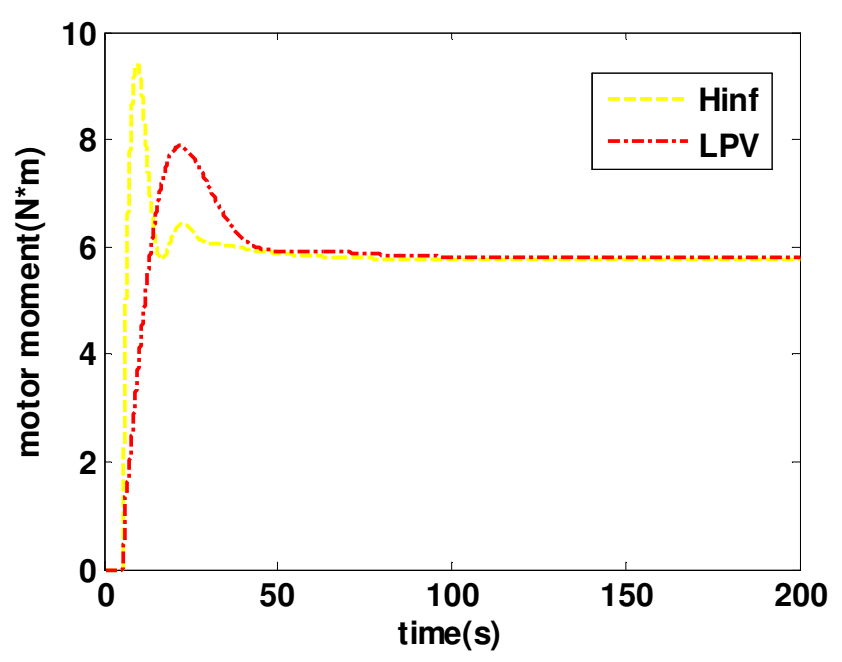

(f)

Notes: (a)pitch angle tracking response, (b)pitch angle tracking error, (c) pitch motor torque, (d) side-slip angle tracking response, (e) side-slip angle tracking error, (f) side-slip motor torque

It can be seen from Figure 5 that the pitch angle tracking error corresponding to the $H_{\infty}$ robust controller is less than 3 per cent and the settling time is $53 \mathrm{~s}$, which can meet the design requirements of the ASS servo system. It shows that when the force- 
displacement gradience is constant, the $H_{\infty}$ robust controller can achieve good tracking performance in pitch angle control; The pitch angle tracking error of the LPV controller is less than 2 per cent and the settling time is $44 \mathrm{~s}$, it shows that when the stick forcedisplacement gradience is constant, the tracking error and the settling time are better than those of $H_{\infty}$ robust controller.

The side slip tracking error corresponding to the $H_{\infty}$ robust controller is less than 3 per cent and the settling time is 130 s, but its overshoot is close to 60 per cent, which cannot be accepted. It indicates that the $H_{\infty}$ robust controller tracking performance cannot be accepted for side-slip angle tracking control when the force-displacement gradience is constant; The side-slip angle tracking error of the LPV controller is less than 1 per cent, the settling time is $105 \mathrm{~s}$ and the overshoot is less than 35 per cent, it indicates that the tracking error, the settling time and the overshoot of the LPV controller are better than those of $H_{\infty}$ robust controller when the SFD is constant.

Comparison of the motor torque, it can be seen that both controllers have the same motor torque output. In addition, the lateral motor torque is little bigger than the longitudinal motor torque when they have the same signal input.

\section{Case 2}

When the SFD changes with proportional to the stick displacement and the maximum variation reach 42.7 per cent of the initial value, the simulation results of the two control strategies are shown in Figure 6. Figure 6(a) shows the pitch angle tracking response under the step signal input, Figure 6(b) is the pitch angel tracking error and Figure 6(c) is the pitch torque output of the motor. Figure 6(d) shows the side-slip angle tracking response under the step signal input, Figure 6(e) is the side-slip angle tracking error, and Figure 6(f) is the side-slip torque output of the motor.

Figure 6 Tracking responses of pitch angle and side-slip angle step input.

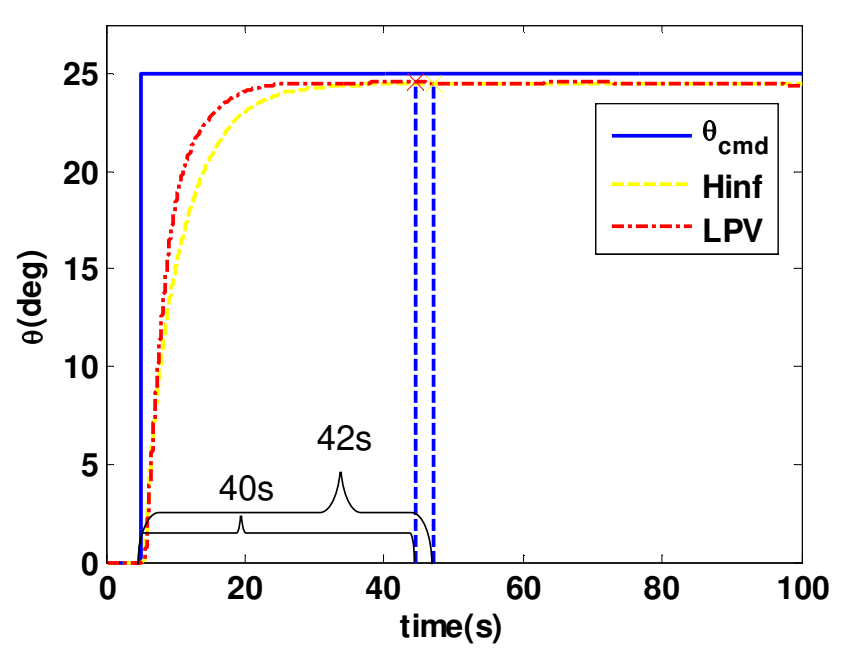

(a)

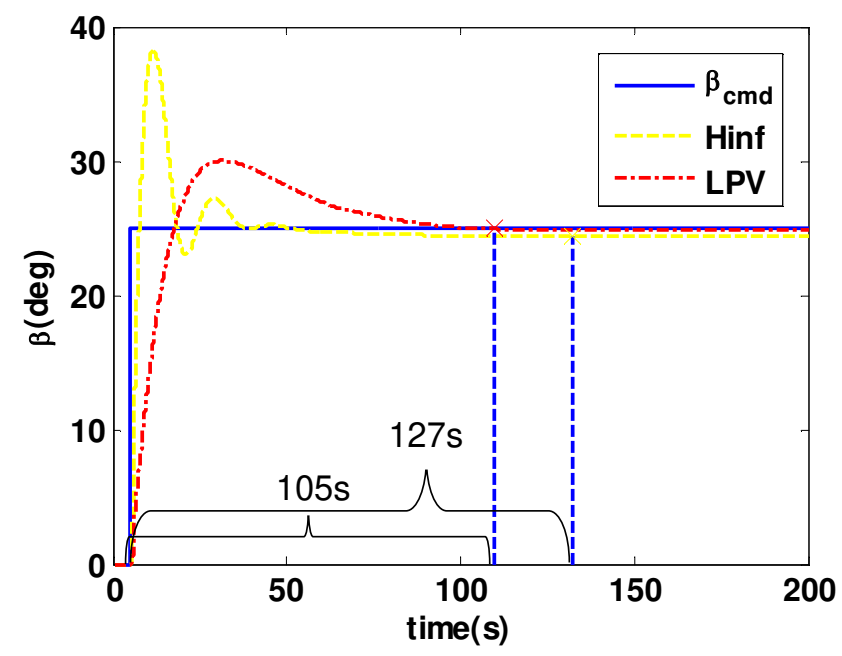

(d) 


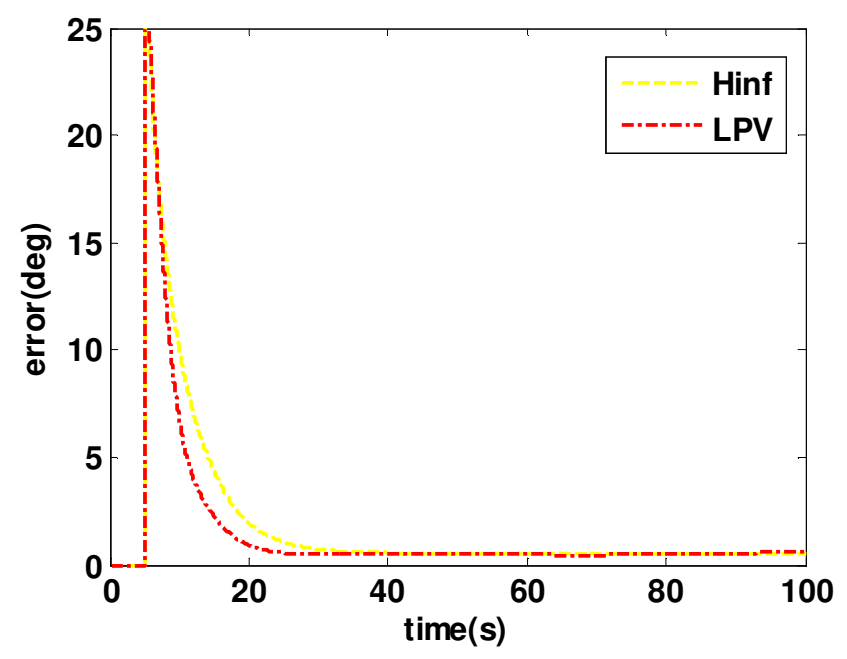

(b)

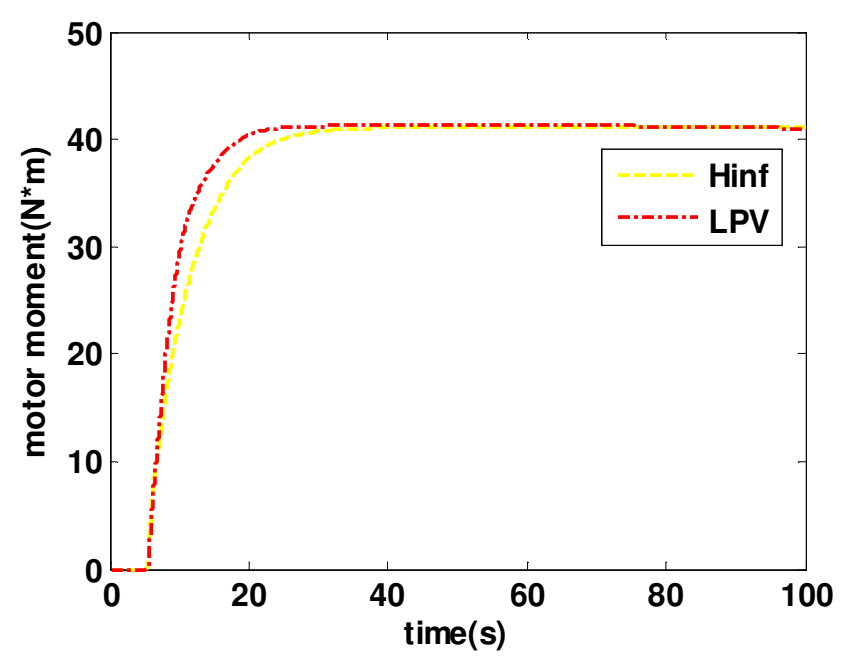

(c)

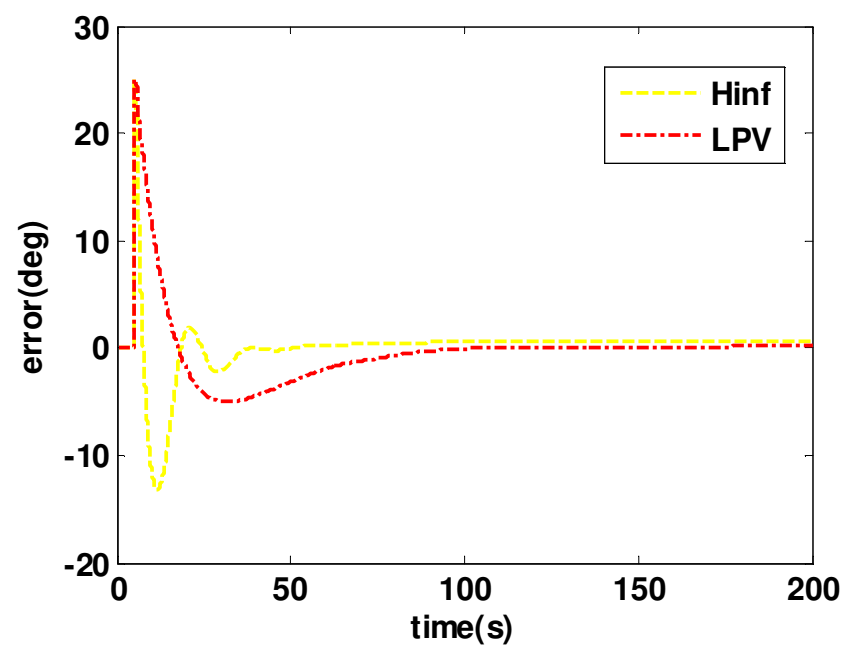

(e)

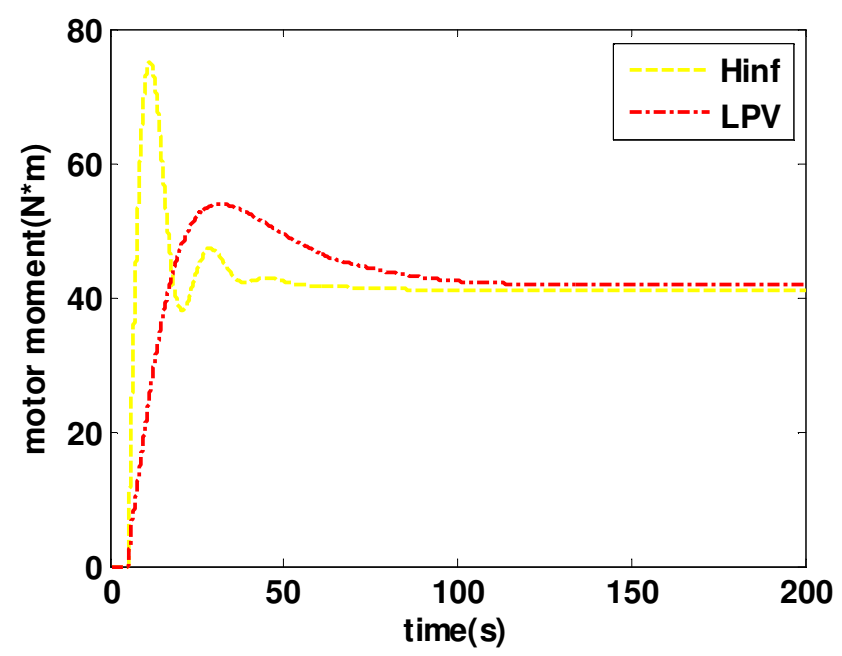

(f)

Notes: (a)pitch angle tracking response, (b)pitch angle tracking error, (c) pitch motor torque, (d) side-slip angle tracking response,

(e) side-slip angle tracking error, (f) side-slip motor torque

It can be seen from Figure 6 that the pitch angle tracking error corresponding to the $H_{\infty}$ robust controller is less than 3 per cent and the settling time is $40 \mathrm{~s}$, which can meet the design requirements of the ASS servo system. It shows that when the SFD gradience changes with proportion to the stick displacement, the $H_{\infty}$ robust controller can achieve good tracking performance in pitch angle control; The pitch angle tracking error of the LPV controller is less than 2 per cent and the settling time is $42 \mathrm{~s}$, the tracking error is better than that of the $H_{\infty}$ robust controller, but the settling time is slightly larger than that of the $H_{\infty}$ robust controller. It indicates that the tracking error of the LPV controller is smaller when the SFD gradience is proportional to the stick displacement, but, as the LPV controller is determined online by gain scheduling, the settling time of the system may be increased.

The side-slip angle tracking error corresponding to the $H_{\infty}$ robust controller is less than 3 per cent and the settling time is $127 \mathrm{~s}$, but its overshoot is up to 55 per cent, which cannot be accepted. It indicates that when the SFD gradience changes with proportional to the stick displacement, the $H_{\infty}$ robust controller tracking performance cannot be accepted in side-slip angle control; The side-slip angle tracking error of the LPV controller is less than 0.5 per cent, the settling time is $105 \mathrm{~s}$ and the overshoot is less than 20 per 
cent, it indicates that the tracking error, the settling time and the overshoot of the LPV controller are better than those of $H_{\infty}$ robust controller when the SFD gradience changes with proportional to the stick displacement.

Comparison of the motor torque, it can be seen that both controllers have the same motor torque output. In addition, the lateral motor torque is the same as the longitudinal motor torque when they have the same input signal.

\section{Case 3}

When the SFD is sinusoidal to the stick displacement and the maximum variation reaches 41.3 per cent of the initial value, the simulation results of the two control strategies are shown in Figure 7. Figure 7(a) shows the pitch angle tracking response under the step signal input, Figure 7(b) is the pitch angle tracking error and Figure 7(c) is the pitch motor torque output. Figure 7(d) shows the side-slip angle tracking response under the step signal input, Figure 7(e) is the side-slip angle tracking error, and Figure 7(f) is the side-slip motor torque output.

Figure 7 Tracking responses of pitch angle and side-slip angle step input.

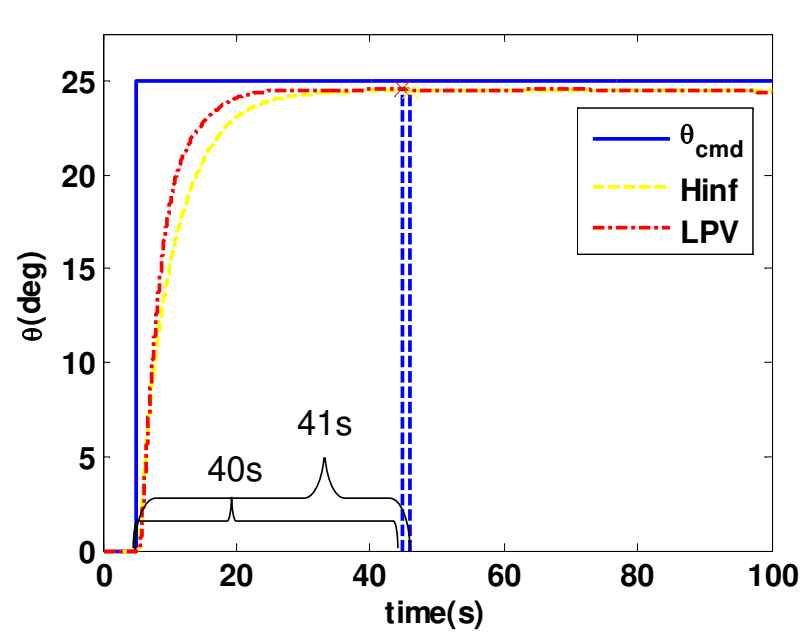

(a)

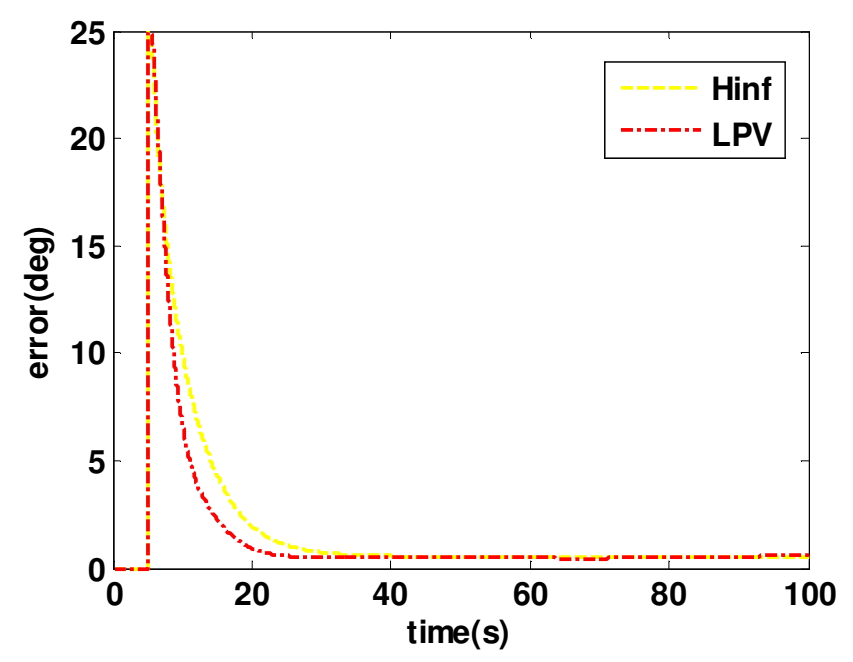

(b)

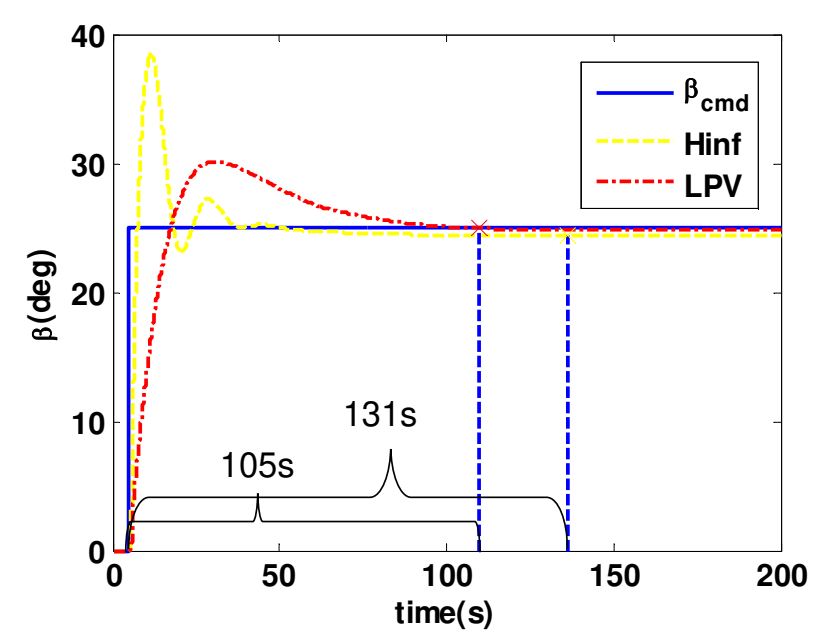

(d)

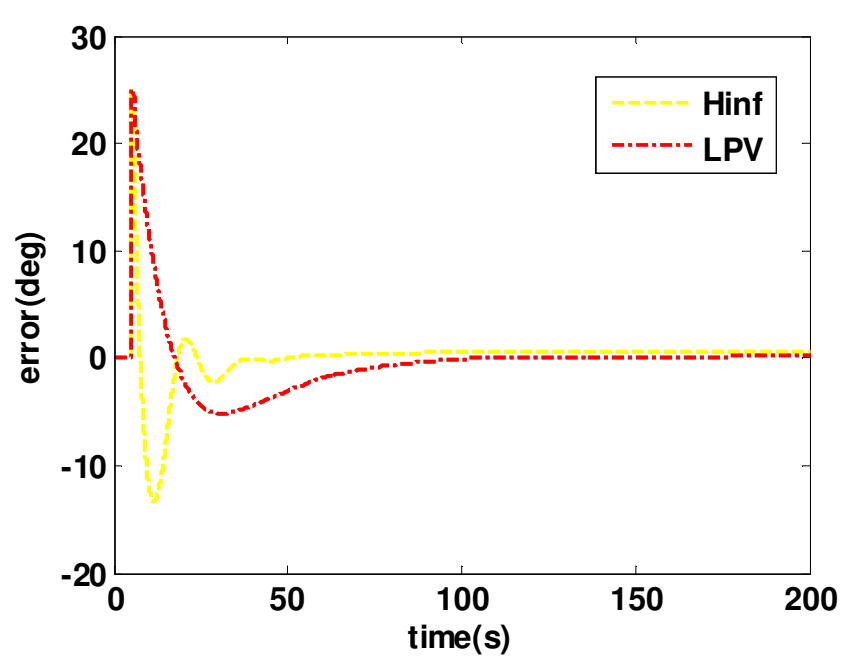

(e) 


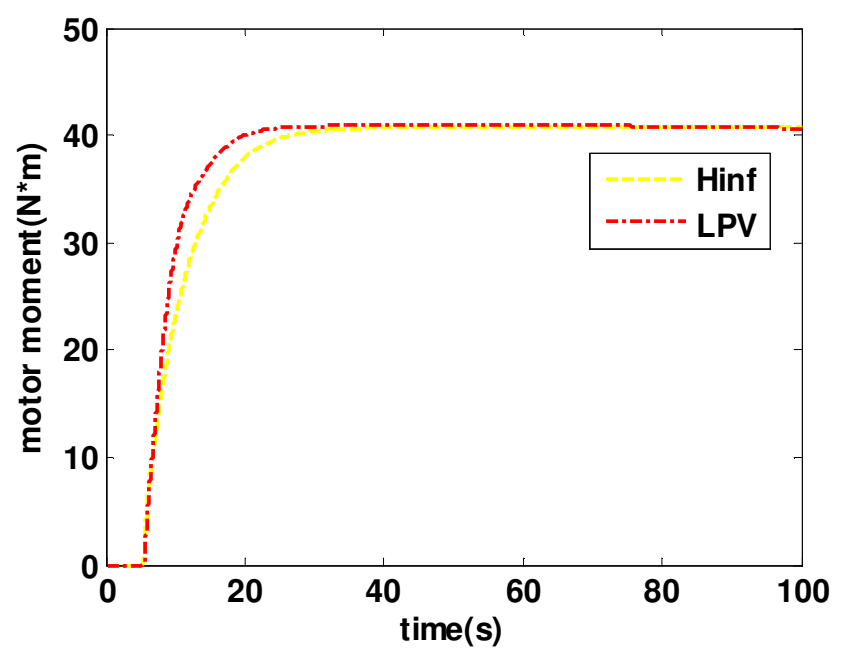

(c)

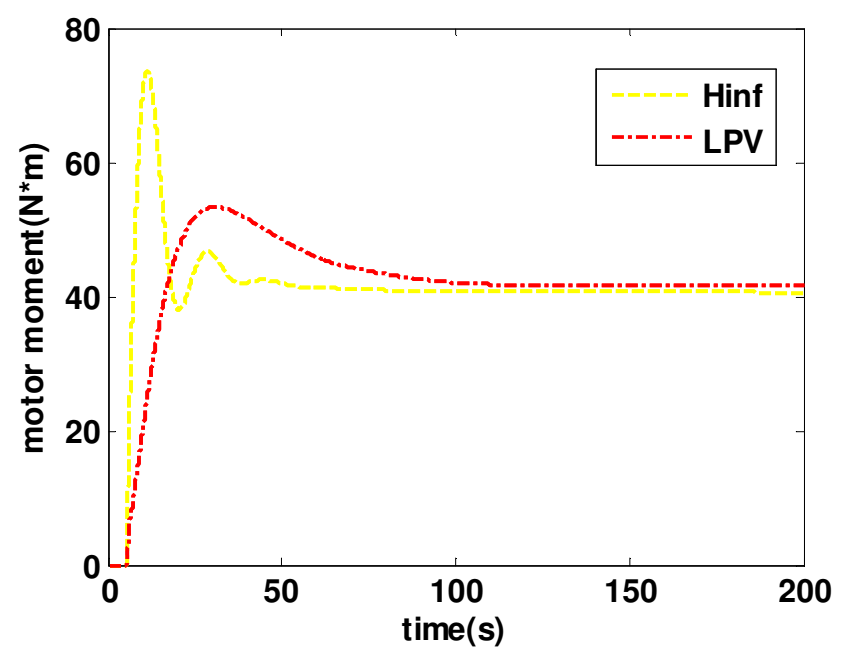

(f)

Notes: (a)pitch angle tracking response, (b)pitch angle tracking error, (c)pitch motor torque, (d) side-slip angle tracking response,

(e) side-slip angle tracking error, (f) side-slip motor torque

It can be seen from Figure 7 that the pitch angle tracking error corresponding to the $H_{\infty}$ robust controller is less than 2.5 per cent and the settling time is $40 \mathrm{~s}$, which can meet the design requirements of the ASS servo system. It indicates that when the SFD gradience is sinusoidal function of the stick displacement, the $H_{\infty}$ robust controller can achieve good tracking performances in pitch angle control; The pitch angle tracking error of the LPV controller is less than 2 per cent and the settling time is $41 \mathrm{~s}$, the tracking error is better than that of the $H_{\infty}$ robust controller, but the settling time is slightly larger than that of the $H_{\infty}$ robust controller. It indicates that the tracking error of the LPV controller is smaller when the SFD gradience is sinusoidal function of the stick displacement, but, as the LPV controller is determined online by gain scheduling, the settling time of the system may be increased.

The side-slip angle tracking error corresponding to the $H_{\infty}$ robust controller is less than 3 per cent and the settling time is $131 \mathrm{~s}$, but its overshoot is up to 55 per cent, which cannot be accepted. It indicates that when the SFD gradience is sinusoidal function of the stick displacement, the $H_{\infty}$ robust controller tracking performance cannot be accepted in side-slip angle control; The side-slip angle tracking error of the LPV controller is less than 0.1 per cent, the settling time is $105 \mathrm{~s}$ and the overshoot is less than 20 per cent, it indicates that the tracking error, the settling time and the overshoot of the LPV controller are better than those of $H_{\infty}$ robust controller when the SFD gradience is sinusoidal to the stick displacement.

Comparing the motor torque, it can be seen that both controllers have the same motor torque output. In addition, the lateral motor torque is the same as the longitudinal motor torque when they have the same input signal.

A comprehensive analysis of the above three cases can lead to the following two conclusions:

1. Comparing Case 1 with Case 2 and Case 3, it can be found that the LPV controller has better tracking performances when the SFD gradience is changing and the tracking error is reduced (Figure 8(a) and Figure 8(c)). Although the settling time will be increased to some extent (Figure $8(\mathrm{~b})$ ) in the pitch angle control, the growth rate is within the acceptable range; by comparing the two control strategies, it shows the tracking performance of the LPV controller is better than that of the $H_{\infty}$ robust controller whenever the SFD gradience changes. The increase of the settling time is acceptable. 
Figure 8 Tracking error and adjustment time comparison figure.

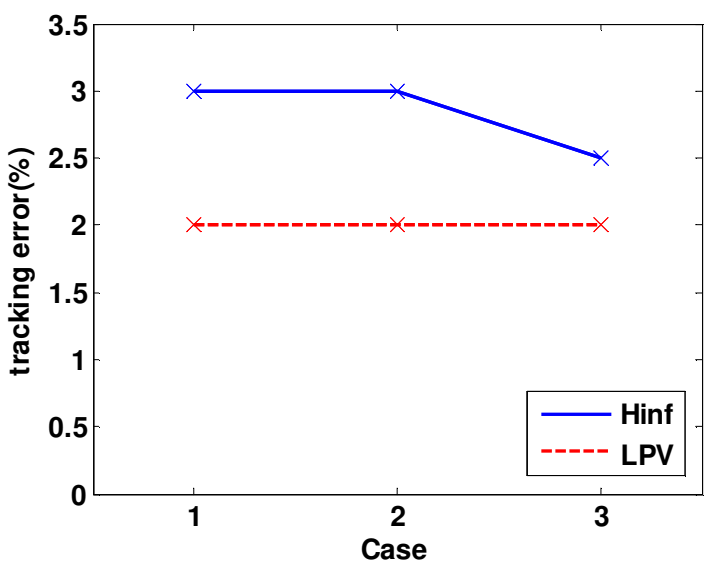

(a)

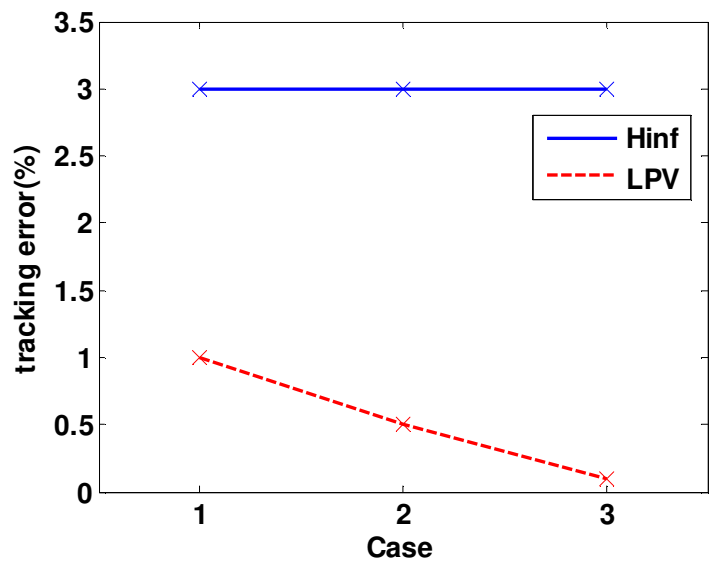

(c)

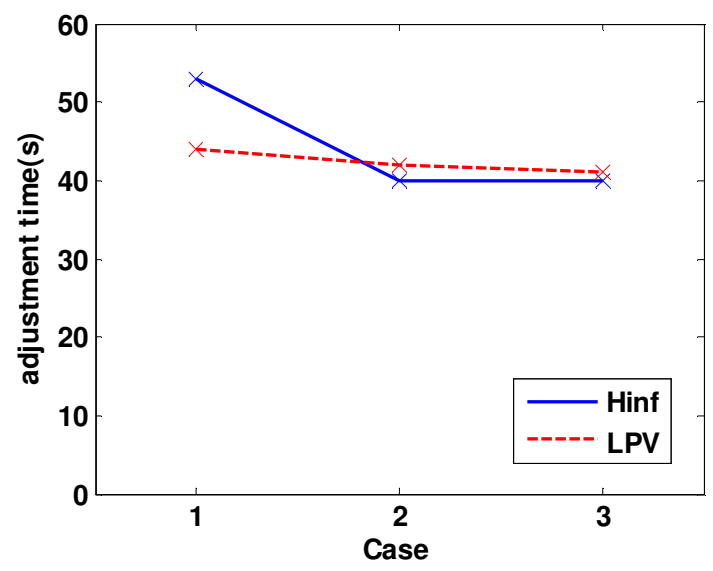

(b)

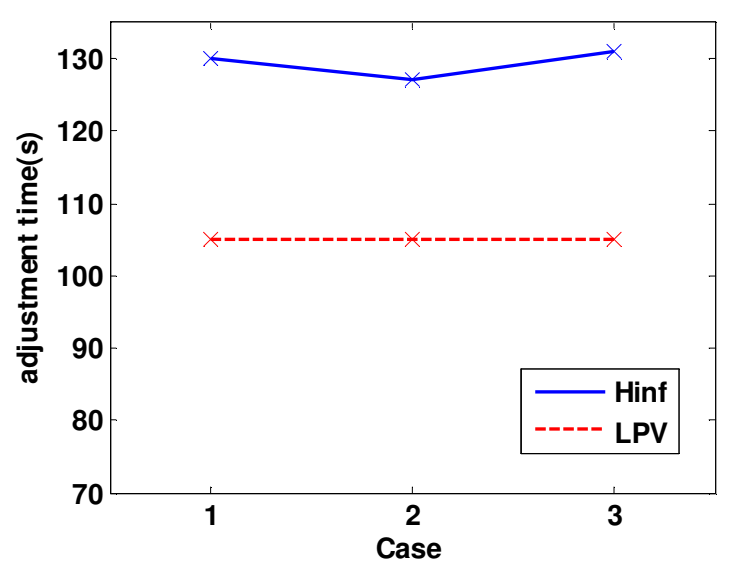

(d)

Notes: (a)pitch angle tracking error, (b)pitch angle adjustment time, (c) side-slip angle tracking error, (d) side-slip angle

\section{tracking settling time}

2. Comparing Case 2 with Case 3, it can be found that the two control strategies can obtain similar results in pitch angle control when the SFD gradience and the stick displacement have different function relationship, it indicates that both control strategies have good robust performances in pitch angle tracking, but LPV controller has better robust performance in sideslip angle tracking and the performance of $H_{\infty}$ robust controller is unacceptable in side-slip angle tracking control.

\section{Conclusion}

In this paper, the problem of the varying SFD gradience is studied and the ASS servo system based on $H_{\infty}$ robust control and LPV control is designed. Based on the Hess pilot model, the simulation model of attitude tracking with the ASS servo system is established. The tracking control of the pitch angle and side-slip angle control is realized by using two control strategies within three cases. By comparing the simulation results, it can be found that LPV control can ensure the robustness of the ASS servo system and it has better tracking performances than $H_{\infty}$ robust control. 


\section{Acknowledgement}

This work has been funded by the National Natural Foundation of China under the Grant 10577102, the Chinese Aviation Science Fund under the Grant 20160757001 and thanks for the anonymous reviewers for their valuable comments and suggestions.

\section{References}

[1] Hosman, R., Benard, B., and Fourquet, H. (1990), “Active and passive side stick controllers in manual aircraft control,” in IEEE International Conference on Systems, Man, and Cybernetics Conference Proceedings, pp.527-529.

[2] Hanlon, C., Potter, C. C., and Wingett, P. T. (2011), Active control stick assembly, USA Patent US 8,056,432 B2.

[3] Feng, H., and Qu, X. J. (2005), “Analysis of the influence of human sense system on human-machine coupling in side stick”, National Annual Meeting of Academic on Flight Mechanics and Flight Test, 2005, Beijing, China, pp. 63-68.

[4] Li, Y. F., and Qu, X. J. (2008), "Method of the human-machine system analysis for the active side stick multi-mode induce," working paper, Beijing University of Aeronautics and Astronautics, Beijing, China.

[5] Liangqing, X. L., and Liu, S. Q. (2017), “Adaptive sliding mode based servo tracking control of aircrafts' active inceptor control systems, ” Journal of Aeronautics, Astronautics and Aviation, Vol.49, No.4, pp. 385-396.

[6] Wei, F., Marinus M., and Max M. (2019), “Developing active manipulators in aircraft flight control,” Journal of Guidance, Control, and Dynamics, Vol.42, No.8, pp. 1755-1767.

[7] Liu, S. Q. (2018), “Flight dynamics and control of modern aircrafts,” Shanghai Jiao Tong University, Shanghai Jiao Tong University Press, Ch. 5, pp. 148-188.

[8] Phillip T. E. (1997), “Modern flight control design, implementation, and flight test," working paper, Air Force Institute of Technology, Dayton, Ohio, USA.

[9] Wei, F., Annemarie L., Marinus M., and Max M. (2018), "Modeling human difference threshold in perceiving mechanical properties from force,” IEEE Transactions on Human-Machine Systems, Vol.48, No.4, pp. 359-368.

[10] Wei, F., and David A. A. (2019), "Framework for human haptic perception with delayed force feedback," IEEE Transactions on Human-Machine Systems, Vol.49, No.2, pp. 171-182.

[11] Gary J. B. (2001), “A linear parameter varying model of the Boeing 747-100/200 longitudinal motion,” working paper, the University of Minnesota, Minnesota, MN.

[12] Subhabrata G. (2003), “Reconfigurable LPV Control Design for a Boeing 747-100/200,” working paper, the University of Minnesota, Minnesota, MN.

[13] Pascal G., and Pierre A. (1994), “A linear matrix inequality approach to $H_{\infty}$ control,” International Journal of Robust and Nonlinear Control, Vol.4, No.4, pp. 421-448.

[14] Gary J. B. (2002), “A linear parameter varying model of the Boeing 747-100/200 lateral/directional motion,” technical report, the University of Minnesota, Minnesota, MN. 
[15] Lu B. (2004), “Linear parameter-varying control of an F-16 aircraft at high angle of attack, " working paper, the Graduate Faculty of North Carolina State University, America.

[16] Shamma, J. S. (1988), “Analysis and design of gain scheduled control systems,” working paper, Massachusetts Institute of Technology, Dept. of Mechanical Engineering, Boston, Massachusetts, USA.

[17] Shamma, J. S., and Athans M. (1991), “Guaranteed properties of gain scheduled control for linear parameter-varying plants,” Automatica, Vol.27, No.3, pp. 559-564.

[18] Apkarian P., Gahinet P., and Becker G. (1995), "Self-scheduled $H_{\infty}$ control of linear parameter-varying systems: a design example," Automatica, Vol.31, No,9, pp. 1251-1261.

[19] Nichols, R. A., Reichert, R. T., and Rugh, W. J. (1993), “Gain scheduling for $H_{\infty}$ controllers: a flight control example,” IEEE Transactions on Control Systems Technology, Vol.1, No.2, pp. 69-79.

[20] Reichert, R. (1992), “Dynamic scheduling of modern-robust-control autopilot designs for missiles,” IEEE Control Systems Magazine, Vol.12, No.5, pp. 35-42.

[21] Hyde, R., and Glover, K. (1993), “The application of scheduled $H_{\infty}$ controllers to a VSTOL aircraft,” IEEE Transactions on Automatic Control, Vol.38, No.7, pp. 1021-1039.

[22] Felix G., and Agnes G. (2018), "Control of longitudinal aircraft motion with loadcase robustness using LPV-control with partly-measurable parameters,” AIAA Guidance, Navigation, and Control Conference, Kissimmee, Florida, USA.

[23] Hess R. A. (1997) “Unified theory for aircraft handling qualities and adverse aircraft pilot coupling," Journal of Guidance Control and Dynamics, Vol. 20, No. 6, pp. 1141-1148.

[24] Hess R A. (2012) “Modeling Pilot Control Behavior with Sudden Changes in Vehicle Dynamics,” Journal of Aircraft, Vol. 46, No. 8, pp. 1584-1592.

[25] Takahiro S., Takashi S., and Hans P. S. (2018), “Robust attitude control using a double-gimbal variable-speed control moment gyroscope,” Journal of Spacecraft and Rockets, Vol.55, No.5, pp. 1235-1247.

[26] Johannes S., Lorenz S., and Walter F. (2018), “Linear parameter-varying control for quadrotors in case of complete actuator loss, ” Journal of Guidance, Control, and Dynamics, Vol.41, No.10, pp. 2232-2242.

[27] Johannes S., and Walter F. (2018), “Gain-scheduled multivariable flight control under uncertain trim conditions,” AIAA Guidance, Navigation, and Control Conference, Kissimmee, Florida, USA. 
2020-03-31

LPV robust servo control of aircraft active side-sticks

Zhou, Guang Rui

Emerald

Zhou GR, Liu SQ, Sang YJ, et al., (2020) LPV robust servo control of aircraft active side-sticks.

Aircraft Engineering and Aerospace Technology, Volume 92, Issue 4, April 2020, pp.599-609

https://doi.org/10.1108/AEAT-08-2019-0155

Downloaded from Cranfield Library Services E-Repository 